A 47-year-old woman was transferred to Harper Hospital for recurrent, severe post-AMI angina refractory to maximal medical therapy, 4 days after sustaining a non-Q-wave inferior AMI associated with a diminutive increase in CK to $300 \mathrm{IU}$. On the day of transfer, she had recurrent rest angina associated with new horizontal ST-segment depression and upright $T$ waves in leads $V_{2}$ through $V_{5}$, believed to represent "remote" anterior subendocardial ischemia or recurrent anterior non-Q-wave AMI (Fig. 2, top left). Blood pressure was $110 / 70 \mathrm{~mm} \mathrm{Hg}$, pulse 72 beats/min and lungs were clear. Urgent coronary angiography showed the left coronary system to be normal. Right coronary artery injection disclosed a high-grade stenotic lesion (at least $95 \%$ luminal diameter narrowing) involving the midportion of the vessel (Fig. 2, top right). Serial inflations with a 2.0-mm Simpson Robert balloon catheter dilated the right coronary artery stenosis (Fig. 2, bottom left). Precordial ST-segment depression abated and abnormal tall $R$ waves did not evolve ( $F$ ig. 2, bottom right).

Recently, we described the early electrocardiographic findings of posterior AMI in 27 patients randomized to the Diltiazem Reinfarction Study of MB-CK-confirmed non-Q-wave AMI. ${ }^{5}$ All patients initially showed a pattern of precordial ST-segment depression with upright $T$ waves, which was believed to represent "anterior" non-Q-wave AMI; however, before discharge, abnormal right precordial $\mathrm{R}$ waves indicative of posterior AMI evolved in all patients.
Coronary angiography was not performed on these study patients by trial design.

We have shown 2 contrasting examples of early posterior AMI due to isolated high-grade 1-vessel disease of a circumflex and right coronary artery, respectively. Each case showed significant early precordial ST-segment depression in the absence of left anterior descending coronary artery disease. These findings emphasize that precordial ST-segment depression with upright $T$ waves due to circumflex or right coronary artery obstruction may produce an early electrocardiographic "current of injury," which is projected as reciprocal precordial ST-segment depression (posterior ST-segment elevation). Such patients may have occluded or subtotally occluded infarct-related coronary arteries, and should not be considered ineligible for acute thrombolylic therapy because of the absence of electrocardiographic ST-segment elevation.

1. Kennedy JW, Ritchie JL, Davis KB, Fritz JK. Western Washington randomized trial of streptokinase in acute myocardial infarction. N Engl J Med 1983:309:1477-1482

2. TIMI Study Group. The Thrombolysis in Myocardial Infarction (TIMI) trial: phase I findings. N Engl J Med 1984;311:932-936.

3. Laffel GL, Braunwald E. Thrombolytic therapy. A new strategy for the treatment of acute myocardial infarction (part I]. N Engl I Med 1984;311:710717.

4. DeWood MA, Stifter WF, Simpson CS, Spures J, Eugster GS, Judge TP, Ninnen ML. Coronary arteriographic findings soon after non-Q wave myocardial infarction. N Engl I Med 1986;313:417-423.

5. Boden WE, Klciger RE, Gibson RS, Schwartz DJ, Schechtman KB, Capone RJ, Roberts R, and the Diltiazem Reinfarction Study Group. Electrocardiographic evolution of posterior myocardial infarction: importance of early precordial ST-segment depression. Am / Cardiol 1987;59:782-787.

\section{Dissolution of Angiographically Detected Intracoronary Thrombus for Unstable Angina Pectoris After Aspirin Therapy}

\author{
ERIC R. BATES, MD \\ JUDITH K. MICKELSON, MD
}

\section{A} therosclerotic plaque disruption with nonocclusive coronary artery thrombosis plays a major role in the pathogenesis of unstable angina pectoris. ${ }^{1}$ Progression to cardiac death or nonfatal acute myocardial infarction (AMI) occurs in more than $10 \%$ of these patients within 3 months $s^{2}$ and is not prevented by conventional medical treatment with nitrate, $\beta$-blocking or calcium antagonist drugs. Two multicenter, randomized, double-blind, placebo-controlled trials have shown a $50 \%$ reduction in mortality and nonfatal AMI rates with aspirin therapy, ${ }^{2,3}$ presumably from inhibition of platelet-dependent thrombus formation. This report provides arteriographic evidence of thrombus dissolu-

From the Division of Cardiology, Veterans Administration Medical Center, University of Michigan, 2215 Fuller Road, Ann Arbor, Michigan 48105. Manuscript received February 13, 1987; revised manuscript received and accepted April 27, 1987. tion in patients with unstable angina whose condition improved on aspirin therapy.

Case 1: A 62-year old man had recurrent postinfarction angina after a non-Q-wave inferior wall AMI. Angina did not recur after aspirin was added to the antianginal regimen of isosorbide dinitrate, metoprolol and diltiazem. Cardiac catheterization revealed $a$ $90 \%$ proximal right coronary artery diameter reduction with associated thrombus (Fig. 1). Repeat catheterization 1 month later revealed a $40 \%$ ulcerated stenosis without thrombus.

Case 2: A 67-year-old man had a 15-minute episode of chest pain associated with inferior ST-segment elevation 10 days after a non-Q-wave inferior wall infarction. Angina did not recur after aspirin was added to the antianginal regimen of isosorbide dinitrate and diltiazem. Cardiac catheterization revealed a $90 \%$ middle right coronary artery diameter stenosis with associated thrombus (Fig. 2). Repeat catheterization 1 month later showed a $20 \%$ ulcerated stenosis without thrombus.

Antiplatelet, anticoagulation and fibrinolytic agents are new medical therapies for unstable angina. These treatment strategies are based on the theory that unstable angina is 1 end of a continuum of acute ischemic syndromes including subendocardial AMI, transmural AMI and ischemic sudden death, which result from atherosclerotic plaque disruption and arterial thrombosis. ${ }^{1}$ Which syndrome develops may depend 


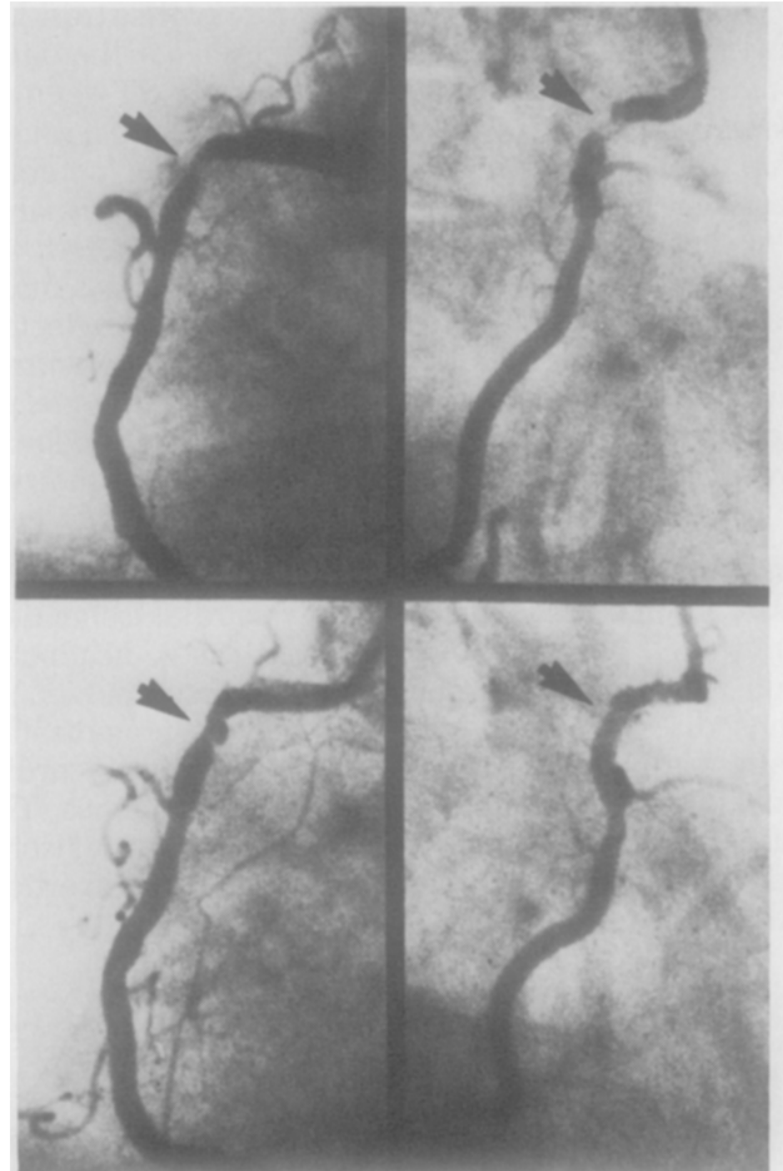

FIGURE 1. Left anterior oblique (left) and right anterior oblique (right) views of a right coronary artery with intracoronary thrombus before treatment (top) and after 1 month of aspirin therapy (bottom). An ulcerated $\mathbf{4 0} \%$ stenosis without thrombus persists.

on the suddenness, completeness and duration of blood flow deprivation caused by platelet thrombi. ${ }^{1}$ Dramatic decreases in mortality and nonfatal AMI have been seen in patients with unstable angina treated with aspirin., ${ }^{23}$ An explanation is that coronary artery thrombosis often occurs adjacent to atherosclerotic plaques of less than $60 \%$ diameter stenosis. ${ }^{4} \mathrm{Be}-$ cause the ischemic syndromes in the 2 patients in this report stabilized on aspirin therapy, we avoided the risks of angioplasty in the setting of angiographically detected intracoronary thrombus. ${ }^{5}$ Cardiac catheterization performed 1 month later in each patient revealed resolution of the thrombus and a widely patent artery. Thus, neither emergency nor elective angioplasty was performed.

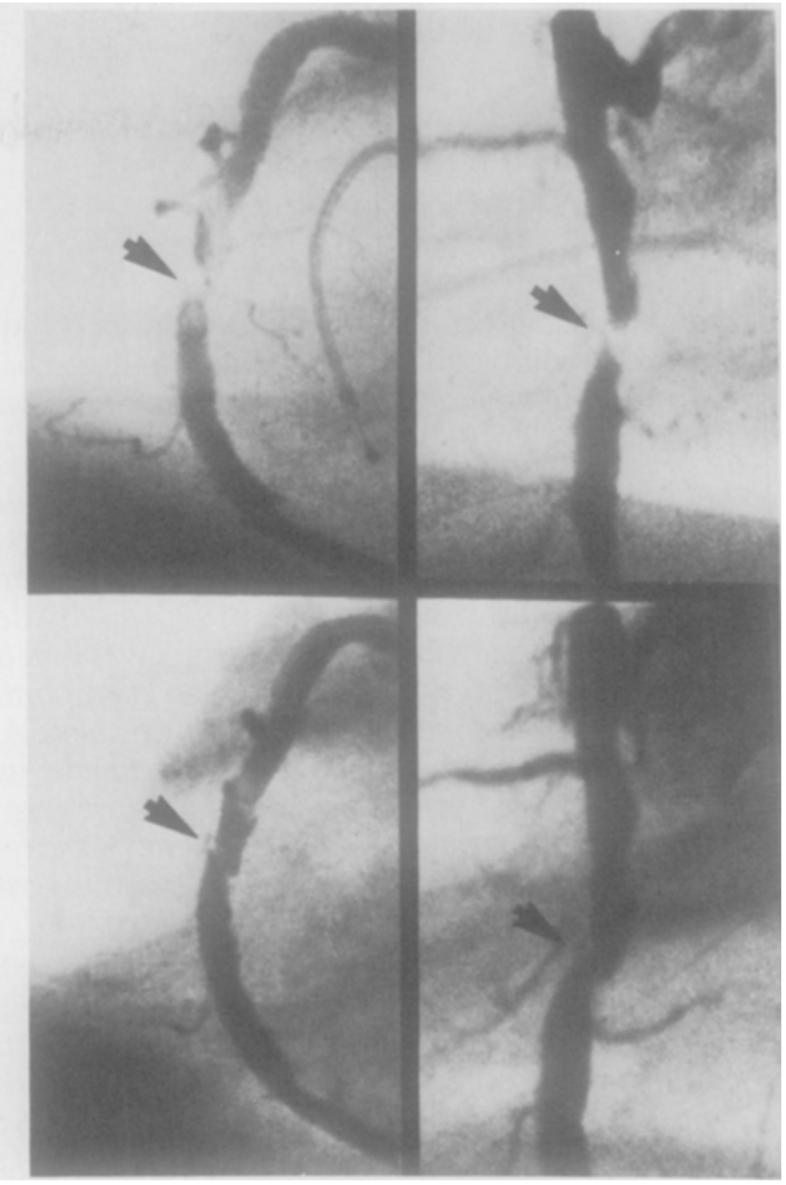

FIGURE 2. Left anterior oblique (left) and left posterior oblique (right) views of a right coronary artery with intracoronary thrombus before treatment (top) and after 1 month of aspirin therapy (bottom). An ulcerated $20 \%$ stenosis without thrombus persists.

1. Gorlin R, Fuster V, Ambrose JA. Anatomic-physiologic links between acute coronary syndromes. Circulation 1986;74:6-9.

2. Lewis HD, Davis JW, Archibald DG, Steinke WE, Smitherman TC, Doherty JE Schnaper HW, LeWinter MM, Linares E, Pouget IM, Sabharwal SC Chesler E, DeMots H. Protective effects of aspirin against acute myocardial infurction and death in men with unstable angina: results of a Veterans Ad ministration cooperative study. $N$ Engl I Med 1983;309:396-403.

3. Cairns JA Gent M, Singer I Finnie KJ Froggatt GM, Holder DA, Jablonsky G, Kostuk WJ, Melendez LJ, Mycrs MC, Sackett DL, Sealoy BJ, Tanser PH Aspirin, sulfinpyrazone, or both in unstable angina: results of a Canadian multicenter trial. N Engl I Med 1985;313:1369-1375.

4. Brown BG, Gallery CA, Badgcr RS, Kennedy JW, Mathey D, Bolson EL, Dodge HT. Incomplete lysis of thrombus in the moderate underlying atherosclerotic lesion during intracoronary infusion of streptokinase for acute myocardial infarction: qunntitotive angingraphis nhservations. Girculation 1986; 73:653-661.

5. Mabin TA, Holmes DR, Smith HC, Vliestra RE, Bove AA, Reeder GS, Chesebro JH, Bresnahan JF, Orszulak TA. Intracoronary thrombus: role in coronary occlusion complicating percutaneous transluminal coronary angioplasty. JACC 1985;5:198-202 